latent vorhanden war, eskalierte der Konflikt zwischen den beiden Supermächten nicht gleich nach Kriegsende, sondern Schritt für Schritt erst ab etwa 1947/48. In der Wahrnehmung des westlichen Lagers spielte - neben der erwähnten Etablierung des sowjetischen Herrschaftssystems in Ost- und Südosteuropa; die Länder wurden später als Satellitenstaaten apostrophiert - die als unmittelbare Bedrohung empfundene kommunistische Expansionspolitik in Richtung auf das übrige Europa die entscheidende Rolle. Im Zentrum des Ost-West-Konfliktes stand aus geopolitischen Gründen Deutschland. Hier prallten die weltanschaulichen Konzepte der beiden Hauptkontrahenten direkt aufeinander. Im Mittelpunkt der Rivalität zwischen den Vereinigten Staaten und der Sowjetunion stand Berlin. Rückblickend schreibt der deutsche Historiker Josef Foschepoth:

Im Zuge der raschen Verschlechterung der Beziehungen zwischen den Mächten nach dem Krieg wurde Deutschland zum eigentlichen Schauplatz jener Auseinandersetzungen, die gemeinhin als Kalter Krieg bezeichnet werden. Aus dem Kampf gegen Deutschland war ein Kampf um Deutschland geworden. Zwar war die Deutsche Frage nicht die Ursache des Kalten Krieges, doch besteht zwischen beiden ein direkter Zusammenhang. ${ }^{104}$

\title{
2.3 Unterschiedliche Vorstellungen der Besatzungsmächte bei der konkreten Deutschlandpolitik
}

Die bedingungslose Kapitulation vom 8. Mai 1945 bedeutete nicht nur das Ende des Naziregimes, sondern hatte auch konsequenterweise den Verlust deutscher Staatlichkeit und mithin deutscher Souveränität zur Folge. Dieses staatsrechtliche Vakuum wurde am 5. Juni 1945 beendet, als die vier Besatzungsmächte - vertreten durch die vier jeweils für ihre Zone zuständigen Oberbefehlshaber General Eisenhower (USA), Marschall Schukow (UdSSR), Feldmarschall Montgomery (Großbritannien) sowie General de Lattre de Tassigny (Frankreich) - in der "Erklärung in Anbetracht der Niederlage Deutschlands« öffentlich bekannt gaben, dass von dem Tage an die oberste Regierungsgewalt in dem Land von den Vertretern der vier alliierten Siegerstaaten übernommen und von ihnen gemeinsam ausgeübt werde. ${ }^{105}$

Tatsächlich sollten jedoch die wichtigsten Fragen in Bezug auf die alliierte Nachkriegspolitik im besetzten Deutschland auf der vom 17. Juli bis zum 2. August 1945 dauernden Potsdamer Konferenz thematisiert und geklärt werden. Die Regierungschefs der drei Siegermächte - die Vereinigten Staaten wurden von dem erstmals auf der internationalen Bühne hervortretenden neuen Präsidenten Harry Truman vertreten - erzielten in einigen Grundsatzfragen eine Übereinkunft. Das in die vier Besatzungszonen aufgeteilte Deutschland - einschließlich Berlins - sollte als eine wirtschaftliche Einheit behandelt werden. »Da außerdem«, so der deutsche Historiker Hermann Graml, »die baldige Errichtung deutscher Zentralbehörden - als Hilfsorgane des Alliierten Kontrollrates - vereinbart wurde, sah es in der Tat so aus, als sei

104 Josef Foschepoth (Hg.), Kalter Krieg und Deutsche Frage. Deutschland im Widerstreit der Mächte 1945-1952, Göttingen/Zürich 1985, Einleitung S. 11-31, hier S. 11, vgl. grundsätzlich hierzu Ernst Nolte, Deutschland und der Kalte Krieg, Stuttgart 1985. 
die Abkehr von den früheren Teilungsplänen endgültig, als werde mithin zumindest das Besatzungsgebiet die politische Einheit bewahren können. «106

Nicht vorgesehen wurde eine zentrale deutsche Regierung. Die gemeinsame Verwaltung der vier Zonen einschließlich der Hauptstadt Berlin sollte durch den Alliierten Kontrollrat erfolgen. Allerdings widersprach die Vorstellung der drei Regierungschefs, Deutschland als wirtschaftliche Einheit zu erhalten, den Beschlüssen zur gemeinsamen Reparationspolitik. Denn es wurde keineswegs eine einvernehmliche Festsetzung einer gesamtdeutschen Reparationsschuld beschlossen, sondern sie verständigten sich darauf, dass "jede Besatzungsmacht ihre Reparationsforderung ohne Einmischung der Partner weitgehend durch direkte Entnahmen aus der eigenen Zone befriedigen dürfe ${ }^{107}$. (Die Sowjetunion sollte zudem 25 Prozent der Demontagen aus den stärker industrialisierten westlichen Besatzungszonen erhalten). Das heißt, nachdem die Wirtschaftseinheit Deutschlands in Potsdam vereinbart wurde, wurde sie durch die »reparationspolitische Teilung« (Theodor Eschenburg) im selben Atemzug gleich wieder infrage gestellt. Die Reparationsabsprache beinhaltete schon das sich langsame Auseinanderentwickeln der Besatzungszonen. Die Vereinigten Staaten und Großbritannien legten sich in Bezug auf die Reparationsansprüche in ihren Zonen Zurückhaltung auf, da sie kein Interesse an einer industriellen bzw. ökonomischen Verelendung hatten.

Für das Auseinanderentwickeln der Besatzungszonen sorgte darüber hinaus die bereits auf den vorangegangenen Konferenzen in Teheran und Jalta angesprochenen Maßnahmen einer Demokratisierungspolitik im okkupierten Deutschland, die sicherstellen sollten, dass dieses Land kein weiteres Mal zu einer Gefahr für Europa bzw. für den Weltfrieden werden könne. Das Potsdamer Abkommen sah vor, neben der Entmilitarisierung des Landes, der industriellen Entflechtung und der strafrechtlichen Verfolgung von Kriegsverbrechern, eine rigorose »Entnazifizierung« von Verwaltung, Bildung, Kultur und öffentlichem Leben, d.h. der gesamten deutschen Gesellschaft, in Gang zu setzen. Damit alle Nationalsozialisten aus dem öffentlichen Leben entfernt werden konnten, sollte durch eine umfassende sogenannte Säuberungsaktion, so Hermann Graml,

die politische Vergangenheit aller erwachsenen Deutschen genau geprüft und eine festgestellte Schuld oder Verstrickung in das NS-Regime je nach Schwere und Grad mit Haft, Berufsverbot oder Geldbußen geahndet werden. Zugleich betonten die Vormünder aber ihre Bereitschaft und sogar Entschlossenheit, auch positive Beiträge zur Demokratisierung der deutschen Cesellschaft zu leisten und den Aufbau demokratischer Institutionen zu fördern. Dabei wollten die Sieger vor allem auf die Wirkung politischer Pädagogik vertrauen: Mit einem umfassenden Erziehungsprogramm müsse der nationalsozialistische Geist überwunden und die Bekehrung der Deutschen zu den Werten, Einrichtungen und Spielregeln der Demokratie erreicht werden. ${ }^{108}$

106 Hermann Graml, Strukturen und Motive alliierter Besatzungspolitik in Deutschland, in: Benz, Deutschland unter alliierter Besatzung, S. 21-32, hier S. 25.

107 Ebd.

108 Ebd., S. 26. 
Die auf den ersten Blick als schlüssiges Demokratisierungsprogramm der Besatzungsbehörden erscheinenden diesbezüglichen Formulierungen im Potsdamer Protokoll ließen indes unberücksichtigt, dass der genauen Begriffsbestimmung des Wortes Demokratie zwischen der Sowjetunion zum einen, Großbritannien und den Vereinigten Staaten zum anderen gänzlich unterschiedliche Vorstellungen zugrunde lagen. Die Komplikationen, die die auf der Potsdamer Konferenz schriftlich formulierten Vereinbarungen zwischen Stalin, Truman und Churchill bzw. dessen Nachfolger Attlee besaßen, wurden langsam sichtbar, als es darum ging, das Demokratisierungsvorhaben der jeweiligen Besatzungsbehörde in die Tat umzusetzen und - wie gesagt - bei der Reparationspolitik Deutschland als wirtschaftliche Einheit zu behandeln. Ein Ergebnis der im Laufe der Zeit aus den verschiedensten Gründen resultierenden Differenzen zwischen den drei bzw. mit Frankreich vier Besatzungsmächten im Alliierten Kontrollrat war die Umsetzung des Mitte 1946 gefassten Beschlusses, die amerikanische und britische Zone territorial zusammenzuschließen; am 1. Januar 1947 erfolgte die Gründung der Bizone.

Der damals als amerikanischer Botschaftsrat in Moskau tätige George F. Kennan formulierte seine Skepsis angesichts der Potsdamer Beschlüsse in einem Entwurf zu einer Denkschrift im Sommer 1945. Hierzu schrieb er:

Die Idee, Deutschland gemeinsam mit den Russen regieren zu wollen, ist ein Wahn. Ein ebensolcher Wahn ist es, zu glauben, die Russen und wir könnten uns eines schönen Tages höflich zurückziehen, und aus dem Vakuum werde ein gesundes und friedliches, stabiles und freundliches Vakuum steigen. Wir haben keine andere Wahl, als unseren Teil von Deutschland - den Teil, für den wir und die Briten die Verantwortung übernommen haben - zu einer Form von Unabhängigkeit zu führen, die so befriedigend, so gesichert, so überlegen ist, daß der Osten sie nicht gefährden kann. ${ }^{109}$

Und Kennan weiter:

Ich kann mich an kein politisches Dokument erinnern, das mich je so deprimiert hätte wie das von Truman unterzeichnete Kommuniqué am Ende dieser wirren und verwirrenden Verhandlungen. Nicht nur weil ich wußte, daß die Idee einer gemeinsamen Viermächtekontrolle, die man jetzt zur Grundlage für die Regierung Deutschlands gemacht hatte, abwegig und undurchführbar sei. Auch die unpräzise Ausdrucksweise, die Verwendung so dehnbarer Begriffe wie »demokratisch«, »friedlich«, »gerecht« in einem Abkommen mit den Russen lief allem direkt zuwider, was siebzehn Jahre Rußlanderfahrung mich über die Technik des Verhandelns mit der sowjetischen Regierung gelehrt hatten. [...] Für die weitere Behauptung, man werde die politische Tätigkeit»demokratischer Parteien und die dazugehörige Versammlungsfreiheit und öffentliche Diskussion« nicht nur gestatten, sondern »ermutigen«, würden mildernde Umstände schwer zu finden sein. Jeder Mensch in Moskau hätte unsern Unterhändlern sagen können, was die sowjetische Führung unter »demokratischen Parteien«verstand. Die Irreführung der Öffentlichkeit in Deutschland und im Westen durch die Verwendung eines solchen Ausdrucks in einem Dokument, das außer von Stalin auch von den Herren 
Truman und Attlee unterzeichnet war, ließ sich selbst mit allergrößter Naivität nicht entschuldigen. ${ }^{110}$

Indes folgte die Deutschlandpolitik der Sowjetunion nach Kriegsende wie in den anderen Ländern seines Macht- und Interessenbereiches in Ost- und Südosteuropa von Anbeginn keiner eindeutig festgelegten Konzeption. Es existierte in dieser Frage seitens der Moskauer Führung kein »Masterplan«, sondern sie bewies - wie auch bereits in der zurückliegenden Außenpolitik - eine hohe Flexibilität. In der manichäisch klar aufgeteilten Welt Stalins existierte weiterhin der fundamentale ideologische Gegensatz zwischen kommunistischen östlichen Staaten und kapitalistischen bzw. imperialistischen westlichen Staaten. Für den sowjetischen Regierungschef waren alle Staaten, die außerhalb des sowjetischen Imperiums und mithin seiner persönlichen Kontrolle lagen, potenzielle Gegner. Die kapitalistischen, demokratisch regierten Länder des Westens waren indes existenzielle Widersacher, mit denen er sich weiterhin in einem fundamentalen ideologischen Kampf befand. Gleichwohl zählte die Vermeidung einer konkreten militärischen Konfrontation mit dem »kapitalistischen Feind« nach dem Sieg gegen den deutschen Nationalsozialismus zu einem zentralen Ziel der stalinschen Politik. Zunächst ging es primär um die Konsolidierung und die Disziplinierung des eigenen Lagers und um den vorsichtigen, schrittweisen Ausbau des Einflusses auch in nicht kommunistisch regierten Ländern. Das schloss in der Vergangenheit keineswegs eine situationsbedingte Kompromisspolitik mit den Partnern der Anti-Hitler-Koalition aus, solange es den sowjetischen Zielen und Interessen zupasskam. Gleichzeitig lag der Fokus in den Ländern Ost- und Südosteuropas auf einer sich langsam durchsetzenden, variablen Politik der Sowjetisierung dieser Länder. Diese Politik musste zum Teil nicht im Widerspruch zu gemachten Konferenzvereinbarungen mit den alliierten Partnern stehen - die Vereinbarungen zum Beispiel von Jalta oder auch von Potsdam waren in einigen Punkten überaus vage und interpretationsbedürftig. Zu Dissonanzen kam es nicht zuletzt aus dem Grund, dass die Moskauer Zentrale alliierte Erklärungen qua ideologischer Prämissen interpretierte und umsetzte. Die zeigte sich an der Deutschlandpolitik der Sowjetunion in der eigenen Zone.

Während des Krieges favorisierte die sowjetische Staatsführung den Plan, nach der totalen Niederlage des Nationalsozialismus Deutschland zu teilen. Im Lauf der Verhandlungen mit den alliierten Partnern zeigte Stalin dann jedoch seine Bereitschaft, nach der bedingungslosen Kapitulation die Spaltung Deutschlands zu verhindern. Der Kremlchef erklärte sich einverstanden, an dem Prinzip der wirtschaftlichen Einheit des Landes mitzuwirken und auf der Basis der in Potsdam getroffenen Vereinbarungen die Verantwortung für die umfassende Demokratisierungspolitik in der sowjetischen Besatzungszone zu übernehmen. Stalin betrieb in der Deutschlandfrage von Anbeginn eine flexible, pragmatische Realpolitik. Das zentrale Ziel bestand darin, die politische und letztlich militärische Integration Deutschlands in das westliche Lager unter der Führung der Vereinigten Staaten zu verhindern. Die Hoffnungen ruhten vor allem darauf, dass die US-Truppen alsbald aus Europa abgezogen würden. Nach Moskauer Interpretation zielten die Postulate wie Entnazifizierung und industrielle Entflechtung auf die Beseitigung der ökonomischen Grundlagen, die überhaupt den Nationalsozialismus ermöglicht hatte. Genauer: Nach kommunistischer Ideologie 
existierte ein Konnex zwischen dem kapitalistischen Gesellschaftssystem und dem sogenannten deutschen Faschismus. Für das sich in den 1930er-Jahren konstituierte System und Regime unter der Führung von Hitler war in den Augen der sowjetischen Führung zwangsläufig die imperialistische Variante des Kapitalismus verantwortlich.

In der Sowjetischen Besatzungszone (SBZ) zeigte sich sehr früh die Tendenz, die dortigen gesellschaftlichen und politischen Bedingungen nach Moskauer Vorstellungen zu verändern. Die sowjetischen Vertreter betrieben eine rücksichtslose und überstürzt vorangetriebene Reparations- und Demontagepolitik. In erster Linie folgte man dem Prinzip, Beute jeder Art zu machen. Wirtschaftlich beuteten sie das Land aus, um für die Kriegsschäden im eigenen Land eine Kompensation zu erhalten. Dies stand zweifelsohne im Einklang mit den Potsdamer Beschlüssen, obwohl sich langfristig die massive Demontage industrieller Eichrichtungen für die Ökonomie in der SBZ mit starken negativen Konsequenzen verband. Gegen den »Geist des Potsdamer Einheitsgebots«, so Hermann Graml, verstieß sie gleichwohl bereits im Sommer 1945, als sie eine Bodenreform einleitete, »die den agrarischen Großgrundbesitz entschädigungslos enteignete, und weitere tiefe Eingriffe in das deutsche Wirtschafts- und Gesellschaftsgefüge « sich bald anschlossen. ${ }^{111}$

Die folgenreichste sowjetische Entscheidung in der SBZ war die Schaffung der Sozialistischen Einheitspartei Deutschlands (SED). Bereits im Juni 1945 konnte sich unter anderem die KPD neu gründen, die aber über ein politisches Schattendasein nicht hinaus kam und isoliert blieb. Um den dann letzten Endes im April 1946 realisierten Zusammenschluss mit der Sozialdemokratischen Partei (SPD) zu ermöglichen, verabschiedete sie sich von dem Ziel »Diktatur des Proletariats«. Als Ziel gab sie an, die »bürgerlich-demokratische Revolution « zu vollenden, und formulierte zu Beginn 1946 die These, dass es einen besonderen deutschen Weg zum Sozialismus geben würde. Vor dem Hintergrund der im Sommer/Herbst 1946 anstehenden Wahlen in den Ländern und Kommunen der SBZ drängte Stalin darauf, die KPD mit der SPD zur SED zu verschmelzen. Die einflussreichsten Positionen wurden mit moskauhörigen Kommunisten besetzt. Alle anderen zugelassenen bürgerlichen Parteien in der SBZ wurden zwar geduldet, waren aber zu einer reinen Statistenrolle verdammt. Die sowjetischen Okkupanten »legten also das Fundament einer Einparteiendiktatur, und dies war mit dem Potsdamer Demokratisierungsauftrag natürlich nur dann vereinbar, wenn man den sowjetischen Demokratiebegriff für richtig hielt ${ }^{112}$.

Die Mehrheit der Bevölkerung in der SBZ erhoffte sich aber ein demokratisches und parlamentarisches System westlichen Zuschnitts, und dies war mit der Monopolisierung der Macht in den Händen einer einzigen politischen Kraft, der SED, nicht vereinbar. Mit der Übertragung des eigenen politischen Systems auf die SBZ verstieß Moskau nicht nur gegen die Beschlüsse der Potsdamer Konferenz, sondern sorgte auch dafür, dass die Bevölkerung der eigenen Zone und der drei anderen Zonen in zunehmenden Maße eine ablehnende Haltung gegenüber der SED und der sowjetischen Besatzungspolitik einnahm.

Mehr und mehr zeichnete sich ab, dass an eine gemeinsame alliierte Deutschland- und Besatzungspolitik nicht zu denken war. Die Spaltung Deutschlands in zwei Lager korrespondierte mit der Verschärfung des Ost-West-Gegensatzes. Nicht zuletzt

111 Graml, Strukturen und Motive alliierter Besatzungspolitik in Deutschland, S. 29.

112 Ebd., S. 30. 
mit der am 1. Januar 1947 erfolgten Vereinigung der amerikanischen und britischen Zone zu einem »Gemeinsamen Wirtschaftsgebiet« wurde der sowjetischen Seite klar, dass ihre Deutschlandkonzeption - in deren Zentrum weiterhin die Reparationspolitik und das Ziel eines Einheitsstaates nach sowjetischem Vorbild standen - zum Scheitern verurteilt war. Denn aus der sogenannten Bizone entwickelte sich fortan ein deutscher Kernstaat und mithin die Integration Westdeutschlands in das von den Vereinigten Staaten dominierte westliche (europäische) System. Sichtbarster Ausdruck für die Unmöglichkeit einer gemeinsamen Besatzungspolitik bzw. für die ideologische Polarisierung der Welt in einen westlichen und östlichen Block war das Scheitern der Moskauer Außenministertagung 1947 und die Ablehnung des Marshallplanes durch die Sowjetunion. Der Plan des amerikanischen Außenministers sah bekanntlich in erster Linie vor, den europäischen Staaten inklusive der osteuropäischen Staaten ökonomische Hilfe zuteilwerden zu lassen. Für Stalin jedoch stellte die ökonomische Einbeziehung der östlichen Staaten in den amerikanischen Regierungsplan eine ernst $\mathrm{zu}$ nehmende Gefahr für den Zusammenhalt der Staaten des sowjetischen Machtbereiches dar. Gleichzeitig wurde mit dem wirtschaftlichen Aufbauplan für Europa der sowjetischen Staatsführung die eindeutige Botschaft vermittelt, dass die amerikanische Regierung keinesfalls daran dachte, sich aus Europa zurückzuziehen. ${ }^{113}$

Mit der Gründung des Kommunistischen Informationsbüros (Kominform) im September 1947, das auf eine verstärkte Kontrolle der kommunistischen Parteien abzielte und die Sowjetisierung des osteuropäischen Staatenblocks beschleunigen sollte, schritt die ideologische Formation der Welt voran. Erstmals erklärte hier der sowjetische Funktionär und Stalin-Abgesandte Andrei Schdanow die politische Leitlinie und verkündete der Weltöffentlichkeit »die These von den beiden einander in unerbittlicher Feindschaft gegenüberstehenden Lagern ${ }^{114}$

\subsection{Die Genese des 'weichen Kurses in der amerikanischen Besatzungspolitik angesichts der sowjetischen Herausforderung und das endgültige Scheitern der diplomatischen Zusammenarbeit der ehemaligen Verbündeten}

Wie gezeigt, wurde in der US-amerikanischen Administration noch während der laufenden Kriegshandlungen ein Planungsprozess in Gang gesetzt, in dem die Frage im Zentrum stand, wie nach dem militärischen Sieg mit dem »deutschen Problem« umzugehen sei. Insbesondere Präsident Roosevelt, der die Gefahren des nationalsozialistischen Expansionskrieges auch für das eigene demokratische Staatswesen sehr früh erkannte, vertrat den eindeutigen Standpunkt, dass nur eine bedingungslose Kapitulation Deutschlands im amerikanischen Interesse sei. Auch unter dem Einfluss seiner Berater stehend, kam er zu dem Entschluss, dass Deutschland kein weiteres Mal Europa mit einem Hegemonialkrieg überziehen darf. Deshalb befürworteten vor der

113 Vgl. exemplarisch zur sowjetischen Deutschlandpolitik in den ersten Nachkriegsjahren zusammenfassend Rolf Badstübner, Die sowjetische Deutschlandpolitik im Licht neuer Quellen, in: Wilfried Frage in der Nachkriegszeit, Berlin 1994, S. 102-135.

114 Cerhard Wettig, Von der Entmilitarisierung zur Aufrüstung in beiden Teilen Deutschlands 19451952, in: Bruno Thoss (Hg.), Vom Kalten Krieg zur deutschen Einheit: Analysen und Zeitzeugenberichte zur deutschen Militärgeschichte 1945 bis 1995, München 1995, S. 3-36, hier S. 8. 